\title{
Graphene: A Nearly Perfect Fluid
}

\author{
Markus Müller, ${ }^{1}$ Jörg Schmalian, ${ }^{2}$ and Lars Fritz ${ }^{3}$ \\ ${ }^{1}$ The Abdus Salam International Center for Theoretical Physics, Strada Costiera 11, 34014 Trieste, Italy \\ ${ }^{2}$ Ames Laboratory and Department of Physics and Astronomy, Iowa State University, Ames, Iowa 50011, USA \\ ${ }^{3}$ Department of Physics, Harvard University, Cambridge, Massachusetts 02138, USA
}

(Received 24 March 2009; revised manuscript received 18 May 2009; published 6 July 2009)

\begin{abstract}
Hydrodynamics and collision-dominated transport are crucial to understand the slow dynamics of many correlated quantum liquids. The ratio $\eta / s$ of the shear viscosity $\eta$ to the entropy density $s$ is uniquely suited to determine how strongly the excitations in a quantum fluid interact. We determine $\eta / s$ in clean undoped graphene using a quantum kinetic theory. As a result of the quantum criticality of this system the ratio is smaller than in many other correlated quantum liquids and, interestingly, comes close to a lower bound conjectured in the context of the quark gluon plasma. We discuss possible consequences of the low viscosity, including preturbulent current flow.
\end{abstract}

DOI: 10.1103/PhysRevLett.103.025301

Graphene [1,2], attracts a lot of attention due to the massless relativistic dispersion of its quasiparticles and their high mobility. Recently, it was shown that this material offers a unique opportunity to observe transport properties of a plasma of ultrarelativistic particles at moderately high temperatures [3]. Undoped graphene is located at a special point in parameter space where the Fermi surface shrinks to two points, and in many respects it behaves similarly as other systems close to more complex quantum critical points [4]. Because of its massless Dirac particles graphene also shares interesting properties with the ultrarelativistic quark gluon plasma. The latter, surprisingly, has an unexpectedly low shear viscosity, as was observed in the dense matter balls created at the relativistic heavy ion collider RHIC [5]. We show here that an analogous property can be found in undoped graphene, reflecting its quantum criticality.

The shear viscosity $\eta$ measures the resistance of a fluid to establishing transverse velocity gradients; see Fig. 1. The smaller the viscosity, the higher the tendency to turbulent flow dynamics. Viscosity, similarly as resistivity in a conductor, leads to entropy production by degrading inhomogeneities in the velocity field. While ideal fluids with $\eta=0$ cannot exist, it is interesting to seek for perfect fluids which come as close as possible to this ideal.

Viscosity has the units of $\hbar n$, where $n$ is some density. To quantify the magnitude of the viscosity, it is natural to compare $\eta / \hbar$ to the density of thermal excitations, $n_{\text {th }}$, which can be estimated by the entropy density, $s \sim k_{B} n_{\text {th }}$. Motivated by the nearly perfect fluid behavior seen in the RHIC experiments, Kovtun et al. have recently postulated a lower bound for the ratio of $\eta$ and $s$ for a wide class of systems [6]:

$$
\eta / s \geq \frac{1}{4 \pi} \frac{\hbar}{k_{B}} .
$$

Equality was obtained for an infinitely strongly coupled conformal field theory by mapping it to weakly coupled
PACS numbers: 05.60.Gg, 71.10. $-\mathrm{w}, 73.23 .-\mathrm{b}, 81.05 . \mathrm{Uw}$

gravity using the AdS-CFT correspondence. While examples violating the bound (1) were found (see [7]), the existence of some lower bound with $k_{B} \eta / \hbar s$ of order unity for a given family of fluids is not unexpected. It is analogous to the Mott-Ioffe-Regel limit for the minimum conductivity of poor metals $[8,9]$, and to the saturation of the relaxation rate at $\tau_{\text {rel }}^{-1}=k_{B} T / \hbar \mathcal{O}(1)$ close to strongly coupled quantum critical points [10]. In all these cases an exhaustion of scattering channels and a saturation of the kinetic coefficients occurs, once the mean free path becomes comparable to the interparticle distance. It follows, that the ratio $\eta / s$ is a unique indicator for how strongly the excitations in a fluid interact.

While effects due to electron-electron interactions only amount to very small additive corrections to the conductivity $\sigma(\omega, T)$ in the collisionless optical regime $\hbar \omega \gg$ $k_{B} T[3,4,11]$, collisions are crucial in the opposite regime $\hbar \omega \ll k_{B} T[3,12]$. There they establish local equilibrium, the remaining low frequency dynamics being governed by
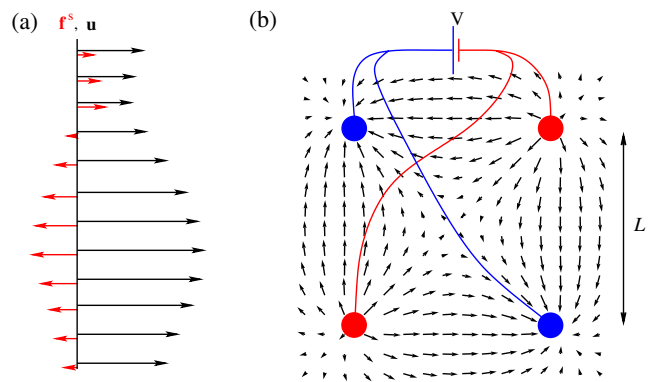

FIG. 1 (color online). (a) Velocity profile $\mathbf{u}$ and associated Stokes force density $\mathbf{f}^{\mathbf{s}}=\eta \nabla^{2} \mathbf{u}$ counteracting the current flow. (b) Inhomogeneous current flow expected in a four-contact geometry with split source and drain contacts held at voltage $\pm V / 2$. In the absence of viscous and other nonlocal effects, the current would be proportional to the applied voltage $V$, independent of the distance $L$ between the contacts. Viscous effects diminish the current as $L$ decreases. 
hydrodynamics, i.e., by the slow diffusion of the densities of globally conserved quantities. Clearly, a hydrodynamic description works best when collisions are frequent and the fluid is strongly correlated. Transport in such a regime reveals information about the nature of the excitations. Examples range from the two He-isotopes [13,14], cold atomic gases $[15,16]$ and electrons in semiconductors and metals $[17,18]$, extending all the way to the high energy regime of the quark gluon plasma [5] and matter in the early universe [19].

In graphene, for energies below a few electron volts, the electronic properties are governed by the Hamiltonian

$$
H=\sum_{l} v \hat{\mathbf{p}}_{l} \cdot \sigma+\frac{1}{2} \sum_{l, l^{\prime}} \frac{e^{2}}{\epsilon\left|\mathbf{r}_{l}-\mathbf{r}_{l^{\prime}}\right|},
$$

with the Fermi velocity $v \simeq 10^{8} \mathrm{~cm} / \mathrm{s}$ [2]. $\hat{\mathbf{p}}_{l}=-i \hbar \nabla_{\mathbf{r}_{l}}$ is the momentum operator, $l=1, \ldots, N$ labels the $N=4$ spin and valley (2 Fermi points) degrees of freedom, and $\sigma=\left(\sigma_{x}, \sigma_{y}\right)$ are the Pauli matrices acting in the space of the two sublattices of the honeycomb lattice structure. Without the Coulomb interaction, Eq. (2) is the Hamiltonian of $N$ species of free massless Dirac particles [20]. The strength of the Coulomb interaction is characterized by the effective fine structure constant $\alpha=\frac{e^{2}}{\epsilon \hbar v} \simeq$ $2.2 / \epsilon$, which is not small for realistic values of the substrate dielectric constant $\epsilon$. Key for an understanding of clean, undoped graphene is the fact that it is a "quantum critical" system with marginally irrelevant Coulomb interactions which renormalize logarithmically to zero $[3,4,11,21-24]$. This quantum critical behavior in undoped graphene is responsible for the distinctly different behavior of the collision-free and collision-dominated frequency regimes [25].

Collision-dominated transport can most efficiently be addressed by solving the Boltzmann transport equation

$$
\left(\frac{\partial}{\partial t}+\frac{1}{\hbar} \frac{\partial \varepsilon_{\mathbf{k} \lambda}}{\partial \mathbf{k}} \cdot \nabla_{\mathbf{x}}-\frac{1}{\hbar} \frac{\partial \varepsilon_{\mathbf{k} \lambda}}{\partial \mathbf{x}} \cdot \nabla_{\mathbf{k}}\right) f=-\mathcal{J}_{\text {coll }}[f]
$$

for the quasiparticle distribution function $f=f_{\lambda}(\mathbf{k}, \mathbf{x}, t)$, which depends on momentum $\mathbf{k}$, position $\mathbf{x}$, time $t$ and band index $\lambda= \pm$ (labeling upper and lower parts of the Dirac cones centered at the two Fermi points). Equation (3) can be derived from a nonequilibrium quantum many body approach, yielding the collision integral $\mathcal{J}_{\text {coll }}$ in terms of the Coulomb interaction [3]. The moments of the distribution function yield the conservation laws for the charge density, $\partial_{t} \rho+\nabla \cdot \mathbf{j}=0$, the momentum density,

$$
\frac{w}{v^{2}}\left[\partial_{t} \mathbf{u}+(\mathbf{u} \cdot \nabla) \mathbf{u}\right]+\nabla p+\frac{\partial_{t} p}{v^{2}} \mathbf{u}+\mathbf{f}^{s}=0,
$$

and the energy density $\varepsilon$. $\mathbf{j}$ is the current density and $\mathbf{u}$ the velocity field of the fluid with enthalpy density $w=\varepsilon+p$, where $p$ is the pressure. For undoped graphene the GibbsDuhem relation implies furthermore $w=T s$. Equation (4) is the Navier-Stokes equation for graphene, derived under the assumption $|\mathbf{u}| \ll v_{F}$. Compared to nonrelativistic hydrodynamics there is an extra relativistic term $\propto \partial_{t} p$, but at low frequencies its effect is small. The Stokes force $f_{j}^{s}=\partial_{i} T_{i j}=\eta \nabla^{2} u_{j}$ is determined by the leading dissipative contribution to the stress tensor:

$$
T_{i j}=\eta X_{i j}+\zeta \delta_{i j} \nabla \cdot \mathbf{u},
$$

in an expansion in gradients of $\mathbf{u}$. Here $X_{i j}=\partial u_{i} / \partial x_{j}+$ $\partial u_{j} / \partial x_{i}-\delta_{i j} \nabla \cdot \mathbf{u}$ corresponds to a pure shear flow while the second term is a volume compression. $\eta$ and $\zeta$ are the shear and bulk viscosity, respectively.

In what follows we include all contributions to $\mathcal{J}_{\text {coll }}$ up to order $\alpha^{2}$, keeping in mind that $\alpha$ flows to zero as $T \rightarrow 0$. Assuming a slowly varying, divergence free velocity field $\mathbf{u}(\mathbf{r})$, we determine the shear viscosity by computing the stress tensor in linear response. Close to equilibrium, an inhomogeneous flow field constitutes a driving term in Eq. (3) of the form $\mathbf{v}_{\mathbf{k}, \lambda} \cdot \nabla_{\mathbf{x}} f_{\lambda}=\sum_{i j} \phi_{i j} X_{j i}$ with

$$
\phi_{i j}(\mathbf{k}, \lambda)=\frac{\varepsilon_{\mathbf{k} \lambda}}{k_{B} T} \frac{e^{\beta \varepsilon_{\mathbf{k} \lambda}}}{2^{3 / 2}\left(e^{\beta \varepsilon_{\mathbf{k} \lambda}}+1\right)^{2}} I_{i j}(\mathbf{k}) .
$$

Here, $\mathbf{v}_{\mathbf{k}, \lambda}=\nabla_{\mathbf{k}} \varepsilon_{\mathbf{k} \lambda} / \hbar$ is the velocity of quasiparticles with energy $\varepsilon_{\mathbf{k} \lambda}, \beta=1 / k_{B} T$ and $I_{i j}(\mathbf{k})=\sqrt{2}\left(\frac{k_{i} k_{j}}{k^{2}}-\frac{1}{2} \delta_{i j}\right)$. In linear response, the distribution function can be parametrized as

$$
\begin{aligned}
f_{\lambda}(\mathbf{k}, t) & =\frac{1}{\exp \left(\beta\left[\varepsilon_{\mathbf{k} \lambda}-\hbar \sum_{i j} X_{i j} g_{j i}(\mathbf{k}, \lambda, t)\right]\right)+1} \\
& \approx f_{\text {eq }}+f_{\text {eq }}\left(1-f_{\text {eq }}\right) \beta \hbar \sum_{i j} X_{i j} g_{j i},
\end{aligned}
$$

where $f_{\text {eq }}=\left.f_{\lambda}(\mathbf{k})\right|_{g_{i j}=0}$. Linearizing the Boltzmann equation in the zero frequency limit, it can be cast into an operator formulation: $|\phi\rangle=\mathcal{C}|g\rangle$ [26,27]. The operator $\mathcal{C}$ is Hermitian with respect to the inner product $\langle a \mid b\rangle=$ $\left(8 \pi^{2}\right)^{-1} \sum_{i j, \lambda} \int d^{2} k a_{i j}(\mathbf{k}, \lambda) b_{j i}(\mathbf{k}, \lambda)$. The $g_{i j}(\mathbf{k}, \lambda)$ parametrize the nonequilibrium distribution function and are obtained by inverting the operator $\mathcal{C}$. Using them to express the stress tensor and comparing with Eq. (5) one obtains the shear viscosity

$$
\eta=\frac{N\left(k_{B} T\right)^{2}}{\sqrt{2} \hbar v^{2}}\left\langle\phi\left|\mathcal{C}^{-1}\right| \phi\right\rangle
$$

The dominant contribution to $\eta$ comes from the smallest eigenvalues of $\mathcal{C}$ restricted to functions given by Eq. (6). The inversion of the collision operator can be a formidable problem and usually requires a numerical solution. The problem simplifies, however, in two dimensions where the amplitude for collinear scattering processes (involving quasiparticles with identical velocity vector) is logarithmically divergent. Screening effects of higher order in $\alpha$ and lifetime effects cutoff this divergence in the infrared at transverse momenta of order $\alpha T / v[3,12]$. To logarithmic accuracy in $\alpha$, we can thus consider collinear scattering processes only. The corresponding restricted operator pos- 
sesses three zero modes:

$$
\begin{gathered}
g_{i j}^{(n)}(\mathbf{k}, \lambda)=c^{(n)} I_{i j}(\mathbf{k}), \quad g_{i j}^{(\chi)}(\mathbf{k}, \lambda)=c^{(\chi)} \lambda I_{i j}(\mathbf{k}), \\
g_{i j}^{(E)}(\mathbf{k}, \lambda)=c^{(E)} \lambda|\mathbf{k}| I_{i j}(\mathbf{k}),
\end{gathered}
$$

which reflect the conservation of charge $n$, chirality $\chi$ (the total number of particles and holes) and energy $E$ in collinear processes. This conservation is exact for the modes $g^{(n, E)}$, while it holds only to lowest order in $\alpha$ for $g^{(\chi)}$, being due to kinetic constraints on the two-body scattering of massless Dirac particles (see [28] for a related discussion). Equation (7) shows that these modes correspond to distribution functions which, when restricted to quasiparticles with identical velocity $\mathbf{v}=v \mathbf{e}$, reduce to equilibria with direction dependent parameters $\mu(\mathbf{e}), \varphi(\mathbf{e}), T(\mathbf{e})$ conjugate to the conserved quantities.

If there were only collinear scattering processes, the shear viscosity would be infinite. However, the inclusion of other processes causes $\eta$ to be finite. Nevertheless, the dominance of collinear scattering allows us-in leading logarithmic approximation - to invert the operator $\mathcal{C}$ within the Hilbert space spanned by the modes (9). At zero doping, a divergence free velocity field does not excite the mode $g_{i j}^{(n)}$, and the relevant subspace is only twodimensional. This inversion is easily done and yields $\left\langle\phi\left|\mathcal{C}^{-1}\right| \phi\right\rangle=C_{\eta} 2^{-3 / 2} \alpha^{-2}$. The remaining numerical coefficient $C_{\eta}$ stems from the evaluation of the matrix elements of the full scattering operator in the $2 d$ subspace of $g_{i j}^{E, \chi}$ and is expected to be of order unity. We obtain $C_{\eta} \simeq$ 0.449 , consistent with this expectation. The $\alpha^{-2}$ dependence follows from the fact that the collision operator $\mathcal{C}$ is of second order in $\alpha$. The shear viscosity of graphene finally results as

$$
\eta=C_{\eta} \frac{N\left(k_{B} T\right)^{2}}{4 \hbar v^{2} \alpha^{2}}\left[1+\mathcal{O}\left(\frac{1}{\log \alpha}\right)\right]
$$

which is the central result of this Letter. It can be rationalized by using the Fermi liquid result [13] $\eta_{\mathrm{FL}} \simeq n m v^{2} \tau_{\text {rel }}$ with $n \rightarrow n_{\text {thermal }} \simeq\left(k_{B} T / \hbar v\right)^{2}$, relaxation rate $\tau_{\text {rel }}^{-1} \simeq$ $k_{B} T /\left(\hbar \alpha^{2}\right)$ [3] and typical energy $m v^{2} \rightarrow k_{B} T$. Extending the analysis beyond the leading approximation by regularizing the logarithmic divergence in the forward scattering and including more modes $g_{i j}$, we obtain corrections of relative size $1 / \log (1 / \alpha)$. For $\alpha=0.1$ they increase the leading result (10) by only $20 \%$.

We need to keep in mind that the quasiparticle are not free, their dispersion reflecting the renormalization of the velocity $v \rightarrow v\left[1+\frac{\alpha}{4} \log (\Lambda / k)\right]$, where $\Lambda$ is an appropriate UV scale. We implement this by a renormalization group approach combined with scaling laws for physical observables. The coupling constant evolves as $\alpha(T) \simeq$ $4 / \log \frac{T_{\Lambda}}{T}$ (with $T_{\Lambda}=\frac{\hbar v \Lambda}{k_{B}}$ ) while the velocity grows logarithmically $v(T)=v \alpha / \alpha(T)$. However, the combination $[\alpha v](T)$ entering $\eta$ does not change under renormalization
[29]. Thus, Eq. (10) is the correct low temperature result for the renormalized quasiparticles.

On the other hand, the entropy density of noninteracting graphene, including renormalization effects, is [4]

$$
s=\frac{9 \zeta(3)}{\pi} k_{B} \frac{k_{B}^{2} T^{2}}{(\hbar v \alpha)^{2}} \alpha^{2}(T) .
$$

The above finally results in the sought ratio:

$$
\eta / s=\frac{\hbar}{k_{B}} \frac{C_{\eta} \pi}{9 \zeta(3)} \frac{1}{\alpha^{2}(T)} \simeq 0.00815 \times\left(\log \frac{T_{\Lambda}}{T}\right)^{2} .
$$

As $T \rightarrow 0$ the ratio $\eta / s$ grows, a behavior expected for a weakly interacting system. However, since $\alpha$ is only marginally irrelevant $\eta / s$ grows only logarithmically. In contrast, in the regime $T \ll \mu$ of doped graphene with a finite carrier density $n$, one obtains the usual behavior of a degenerate Fermi liquid [13,18] with $\eta \sim \hbar n(\mu / T)^{2}$ and $s \sim k_{B} n T / \mu$, in which case $\eta / s \sim\left(\hbar / k_{B}\right)(\mu / T)^{3}$ diverges much more strongly at low $T$; see Fig. 2 . Higher order corrections of the long range Coulomb interaction [24] mainly reduce the regime where $\alpha(T)$ decreases logarithmically (they effectively reduce $T_{\Lambda}$ ). This further decreases the ratio $\eta / s$. Similarly, additional short range interactions $g$ yield leading additive corrections $\propto \alpha g\left(T / T_{\Lambda}\right)$ to the collision operator. Their effect is small provided that they do not lead to an excitonic insulator and the low energy physics of massless Dirac particles is preserved [30].

Note the small numerical prefactor in (12). As shown in Fig. 2, it keeps the ratio $\eta / s$ small in a large temperature regime, where it approaches the value of Eq. (1). As was shown recently, cold atoms with diverging scattering length are materials which also come close to the value (1) $[15,16]$. Our result shows that, interestingly, graphene has an even smaller ratio $\eta / s$, thus being an even "more perfect" liquid than those critical systems. We envision

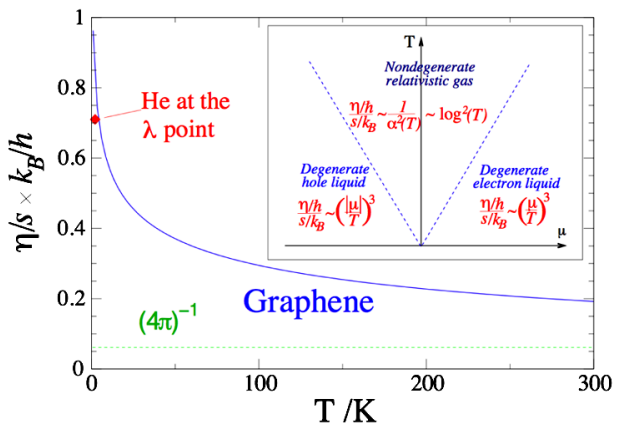

FIG. 2 (color online). Ratio $\eta / s$ in graphene as a function of $T$. The UV cut-off was taken to be $T_{\Lambda}=8.34 \times 10^{4} \mathrm{~K}$ following [4]. In the undoped, quantum critical system $\eta / s$ is very small in a large temperature window where the coupling $\alpha(T)$ remains of order $O(1)$. The value $1 / 4 \pi$ obtained for some strongly coupled critical theories is shown for comparison. As shown in the inset, away from zero doping and quantum criticality $(T<|\mu|)$, the viscosity assumes the behavior of a degenerate Fermi liquid, $\eta / s \sim(|\mu| / T)^{3}$. 
interesting experimental manifestations of viscous effects in graphene, especially in the conductance properties of very clean samples. Viscous drag should result in a decrease of the conductance with the linear size of geometries such as in Fig. 1, where two spatially separated contacts take the role of the source and the drain, respectively. Applying a source-drain bias $V$ to these split contacts induces an inhomogeneous current flow and corresponding Stokes forces that oppose it. The smaller the spatial scale $L$, the larger the viscous forces, and hence one expects an increasing resistance. The latter would be scale invariant in the absence of viscous forces and other nonlocal effects on conductivity [31].

The unusually low viscosity in graphene suggests the interesting possibility of electronic turbulence in this material. For simplicity we consider fluid velocities small compared to the Fermi velocity, and analyze the low frequency limit of the Navier-Stokes Eq. (4). Turbulence arises from the nonlinearities $\propto(\mathbf{u} \cdot \nabla) \mathbf{u}$ while dissipation due to the Stokes forces suppress turbulent flow for large $\eta$. The dimensionless number determining the relative strength of these two effects is the Reynolds number,

$$
\operatorname{Re}=\frac{s / k_{B}}{\eta / \hbar} \frac{k_{B} T}{\hbar v / L} \frac{u_{\mathrm{typ}}}{v},
$$

where we assumed a typical fluid velocity $u_{\mathrm{typ}}$ and a characteristic length scale $L$ for the velocity gradients. Thus, the ratio $\eta / s$ reveals itself as the key characteristic determining the Reynolds number, apart from geometrical factors, typical energies and velocities. To observe turbulence one needs $\operatorname{Re}>10^{3} \cdots 10^{4}$ in $3 d$, and somewhat higher values in $2 d$. However, even for lower $\mathrm{Re} \simeq$ $10 \cdots 10^{2}$ two-dimensional flow in the presence of extended defects or nanosized obstacles undergoes complex phase locking phenomena and chaotic flow [32]. Applying strong bias fields to graphene, fluid velocities of the order $u_{\mathrm{typ}} \simeq 0.1 v$ can be achieved [33], while still avoiding the onset of non-Ohmic effects. In the collision-dominated regime of undoped graphene the fluid velocity induced by a field $E$ scales like $u / v \sim e E \hbar v /\left(k_{B} T\right)^{2}$. Hence, the Reynolds number increases as $1 / T$ with decreasing $T$, enhancing the tendency towards turbulence. With flow velocities as estimated above we expect complex fluid dynamics as in Ref. [32] already on small length scales of the order of $L \sim 1 \mu \mathrm{m}$. This would constitute a striking manifestation of the quantum criticality of graphene and could be relevant for potential nanoelectronics applications of this exciting material.

We thank S. Hartnoll, D. Nelson and S. Sachdev for useful discussions. M. M. and J.S. acknowledge the hospitality of the Aspen Center for Physics. The authors were supported by the SNF under grants PA002-113151 and PP002-118932 (M.M.), the Ames Laboratory, operated for the U.S. DOE by Iowa State University under Contract No. DEAC02-07CH11358 (J. S.), and DFG grant Fr 2627/1-1 and NSF grant DMR-0757145 (L.F.).
[1] K. S. Novoselov, A. K. Geim, S. V. Morozov, D. Jiang, Y. Zhang, S.V. Dubonos, I. V. Grigorieva, and A. A. Firsov, Science 306, 666 (2004).

[2] K. S. Novoselov, A. K. Geim, S. V. Morozov, D. Jiang, M. I. Katsnelson, I. V. Grigorieva, S. V. Dubonos, and A. A. Firsov, Nature (London) 438, 197 (2005).

[3] L. Fritz, J. Schmalian, M. Müller, and S. Sachdev, Phys. Rev. B 78, 085416 (2008).

[4] D.E. Sheehy and J. Schmalian, Phys. Rev. Lett. 99, 226803 (2007).

[5] E. Shuryak, Prog. Part. Nucl. Phys. 53, 273 (2004).

[6] P. Kovtun, D. T. Son, and A. O. Starinets, Phys. Rev. Lett. 94, 111601 (2005).

[7] Y. Kats and P. Petrov, J. High Energy Phys. 01 (2009) 044; A. Buchel, R.C. Myers, and A. Sinha, J. High Energy Phys. 03 (2009) 084.

[8] A.F. Ioffe and A.R. Regel, Prog. Semicond. 4, 237 (1960).

[9] N. Mott, Philos. Mag. 26, 1015 (1972).

[10] S. Sachdev, Quantum Phase Transitions (Cambridge University Press, Cambridge, England, 1999).

[11] I. F. Herbut, V. Juričić, and O. Vafek, Phys. Rev. Lett. 100, 046403 (2008).

[12] A. B. Kashuba, Phys. Rev. B 78, 085415 (2008).

[13] A. A. Abrikosov and I. M. Khalatnikov, Rep. Prog. Phys. 22, 329 (1959).

[14] I. M. Khalatnikov, An Introduction to the Theory of Superfluidity (Benjamin, New York, 1965).

[15] G. M. Bruun and H. Smith, Phys. Rev. A 75, 043612 (2007).

[16] T. Schäfer, Phys. Rev. A 76, 063618 (2007).

[17] G. Vignale, C. A. Ullrich, and S. Conti, Phys. Rev. Lett. 79, 4878 (1997).

[18] M. S. Steinberg, Phys. Rev. 109, 1486 (1958).

[19] S. Weinberg, Astrophys. J. 168, 175 (1971).

[20] P. R. Wallace, Phys. Rev. 71, 622 (1947).

[21] J. González, F. Guinea, and M. A. H. Vozmediano, Nucl. Phys. 424B, 595 (1994); Phys. Rev. B 59, R2474 (1999).

[22] J. Ye and S. Sachdev, Phys. Rev. Lett. 80, 5409 (1998).

[23] E. V. Gorbar, V.P. Gusynin, V. A. Miransky, and I. A. Shovkovy, Phys. Rev. B 66, 045108 (2002).

[24] D. T. Son, Phys. Rev. B 75, 235423 (2007).

[25] K. Damle and S. Sachdev, Phys. Rev. B 56, 8714 (1997).

[26] P. Arnold, G. D. Moore, and L. G. Yaffe, J. High Energy Phys. 11 (2000) 001.

[27] J. M. Ziman, Electrons and Phonons (Oxford University Press, Oxford, 1960), Chap. 7.

[28] M. S. Foster and I. L. Aleiner, Phys. Rev. B 79, 085415 (2009).

[29] M. P. A. Fisher and G. Grinstein, Phys. Rev. Lett. 60, 208 (1988); I. F. Herbut, Phys. Rev. Lett. 87, 137004 (2001).

[30] I. F. Herbut, Phys. Rev. Lett. 97, 146401 (2006).

[31] D. A. Abanin and L. S. Levitov, Phys. Rev. B 78, 035416 (2008).

[32] A. K. Saha, K. Muralidhar, and G. Biswas, J. Engin. Mech. ASCE 126, 523 (2000).

[33] I. Meric, M. Y. Han, A. F. Young, B. Ozyilmaz, P. Kim, and K. L. Shepard, Nature Nanotech. 3, 654 (2008). 